\title{
LA INTEGRACIÓN AL ÁMBITO LABORAL DEL PEDAGOGO DE LA UNIVERSIDAD PAN AMERICANA
}

María Teresa Carreras Lomelí con la colaboración de Evelia Vásquez H

\section{INTRO DUCCIÓN}

A 30 años del inicio de la licenciatura en Pedagogía de la Universidad Panamericana,* de la cual han egresado 27 generaciones con un total de 1520 exalumnos, la Facultad de Pedagogía lleva al cabo una reflexión sobre los logros alcanzados en estos años, así como los retos que enfrenta en un futuro inmediato.

La carrera de Pedagogía de la Universidad Panamericana fue creada en 1967 para responder a la necesidad de formación de pedagogos que incidieran en la educación, principalmente en el ámbito escolarizado, en aquella época los profesores normalistas no tenían acceso a la UNAM, por lo cual el IPH ofrecía la alternativa de cursar un año propedéutico y luego iniciar la licenciatura con incorporación UNAM.

En los 30 años de la carrera de Pedagogía han habido tres planes de estudio, los cuales se fueron actualizando y modificando con la finalidad de proporcionar a los alumnos mejor formación humana y profesional.

El primer plan de estudios 67 - 76 estaba conformado por cinco áreas: filosófica, didáctica, psicológica, sociológica e instrumental, en este plan se hacía mayor hincapié en el área didáctica, la cual contaba con el mayor número de materias, esto con la finalidad de ofrecer a los alumnos, los elementos necesarios para desarrollar su trabajo en el ámbito escolar.

En 1977 se dio la primera modificación importante en el plan de estudios al incluir el área administrativa con las siguientes materias: administración básica, teoría de la empresa, enfoque 
económico de la empresa, factor humano, administración de personal, capacitación y desarrollo y política de la empresa. Estas materias se impartían como adicionales al plan de estudios establecido por la UNAM.

En 1986 se realizan algunas modificaciones al plan de estudios con la finalidad de actualizar algunos contenidos, aparece por primera vez tecnología educativa (computación) y orientación familiar en tercer semestre, y se fortalece el área filosófica con materias internas como fundamentos ideológicos de occidente, principios morales de la conducta, metafísica y síntesis del conocimiento teológico.

En 1993* se vuelven a hacer ajustes con miras a la implantación de un nuevo plan de estudios; las áreas instrumental y administrativa sufren modificaciones en su estructura y cambia de nombre a logotécnica y empresarial. el plan de estudios que se llevó al cabo ese año fue un plan de transición que incluía materias tanto del plan UNAM como del nuevo plan de estudios con reconocimiento de validez oficial por parte de la SEP.

En 1994 después de tres años de estudio y análisis de los planes y programas de Pedagogía así como de consultas con especialistas y entrevistas con exalumnos, se inicia el nuevo plan de estudios cuyo objetivo general es:

La formación conceptual operacional de profesionales de la educación que sean capaces de tomar decisiones para diagnosticar, planear, realizar y evaluar acciones que contribuyan a la mejora personal y social, en cualquier etapa evolutiva en los ámbitos empresarial, comunitario, familiar y escolar.

Desde el primer plan de estudios en 1967 al vigente 1994 siempre se han considerado las bases filosóficas que sustentan el quehacer del pedagogo de la Universidad Panamericana con la finalidad de formar no sólo buenos profesionales de la educación sino buenas personas que reflejen en su vida cotidiana la solidez de sus principios. 


\section{UN ACERCAMIENTO A LOS EGRESADOS}

Al llevar al cabo la reflexión sobre el papel de la Facultad en la formación de profesionales nos preguntamos ¿cuántos de nuestros egresados están trabajando ¿dónde? ¿consideran que la Universidad Panamericana les ofreció una buena formación? ¿ésta les ha servido en su vida personal y profesional? ¿cuáles son los elementos de formación que no se les proporcionaron? ¿que les han hecho falta en su ejercicio profesional?

Para dar respuesta a estas interrogantes se diseñó una investigación cuyos objetivos son:

* Obtener información sobre el desempeño profesional del pedagogo de la Universidad Panamericana.

* Conocer la opinión de los egresados con respecto a la formación profesional y humana que recibieron en la Universidad Panamericana.

\section{METODOLOGÍA}

La investigación que se presenta es de tipo descriptivo en su forma de encuesta con modalidad de cuestionario mixto.

Los sujetos de estudio son los egresados de la Universidad Panamericana de 1971 a 1998.

De los 1596 egresados que conforman el universo total se localizaron a 450 los cuales forman la muestra de estudio.

Se enviaron por correo y fax 450 cuestionarios de los cuales solo se recuperaron 106, por lo cual el universo y la muestra quedó configurada de la siguiente manera:

Universo total:

Muestra teórica

Muestra real
1520

450

106

\section{VARIABLES DE ESTUDIO}

Las variables de estudio que dan sustento al cuestionario son las siguientes: 
* Datos generales

* Lugar de trabajo

* Puesto que desempeña

* Sueldo

* Formación académica

* Integración al ámbito laboral.

\section{ELABORACIÓN DE LOS DATOS}

El procesamiento de la información obtenida por medio de cuestionarios se efectuó de la siguiente manera:

* Concentración de los cuestionarios

* Verificación de datos de identificación de cada uno

* Revisión y selección de cada alumno

* Vaciado de resultados a cuadros de concentración

* Elaboración de gráficas

\section{PRESENTACIÓN DE RESULTADOS}

De los exalumnos que respondieron al cuestionario encontramos que el 7\% pertenece a generaciones 71 - 75 a 76 - 80 los cuales fueron formados con el primer plan de estudios que, como ya se señaló hacia énfasis en el área didáctica y materias de cultura general.

El 26\% de las respuestas corresponden a las generaciones 77 - 81 a 86 - 90 formadas con el nuevo plan de estudios que incluye materias administrativas, aunque el enfoque sigue siendo didáctico principalmente.

El 40\% de los exalumnos que respondieron pertenecen a las generaciones 87 - 91 a 92- 96, el plan de estudios que se llevó al cabo en estas fechas presentó modificaciones importantes orientadas a su actualización y por último el $27 \%$ de los egresados que respondieron pertenecen a las generaciones 93 - 97, la última con incorporación a la UNAM la cual llevó el plan de estudios de transición que ya incluía la reestructuración de las 
áreas logotécnica y empresarial y la generación 94 - 98 primera con reconocimiento SEP.

En cuanto a los resultados referentes a la variable trabajo encontramos que el $85 \%$ de nuestros egresados trabajan actualmente, la mayoría de éstos $88 \%$ se desempeñan como pedagogos ubicados casi todos en el sector privado 93\%, distribuidos de la siguiente manera: el 53\% labora en el ámbito escolar, el $32 \%$ en el ámbito empresarial, el $9 \%$ en el ámbito comunitario y el $6 \%$ en el ámbito familiar.

Estos resultados eran previsibles puesto que la mayoría de los egresados que respondieron se formaron con los planes de estudio con incorporación UNAM que hacían más énfasis en el área didáctica y la educación escolarizada.

En relación a la variable puesto que desempeña, horario y salario, encontramos que el $33 \%$ se desempeñan como coordinadores de área, el 14\% tienen un puesto directivo, el $11 \%$ trabajan como gerentes o ejecutivos, el $12 \%$ se dedica solo a la docencia en los diferentes niveles educativos, como auxiliares de directivos y coordinadores encontramos al 10\%, como orientadores se desempeñan el $6 \%$ y el $11 \%$ cae en el rubro de otro que abarca diferentes actividades desarmolladas por una solo persona. El 3\% se dedica a tareas administrativas.

En cuanto al horario y sueldo encontramos que el $66 \%$ trabaja de tiempo completo, percibiendo un salario entre $\$ 6$ 000.00 y $\$ 10000.00$. Los exalumnos que trabajan medio tiempo $22 \%$ perciben un salario entre $\$ 3000.00$ y $\$ 6000.00$ y por último los que trabajan tiempo parcial $12 \%$ su promedio de ingreso está también entre $\$ 3000.00$ y $\$ 6000.00$. Entre los que trabajan tiempo completo encontramos un $17 \%$ que sus ingresos están entre $\$ 10000.00$ y $\$ 15000.00$ pesos y a un $10 \%$ de exalumnos que sus ingresos son mayores a $\$ 15000.00$ porcentajes muy similares a los encontrados en el puesto de gerentes y directivos. 
Pasando a la variable integración al campo laboral encontramos que el 69\% considera que la carrera le ofreció elementos suficientes para su desempeño laboral, entre los elementos que aportó la carrera a su formación están los proporcionados por el área didáctica 28\%, el área filosófica 27\%, el 14\% corresponde al área empresarial y el $17 \%$ tiene que ver con su formación personal, en este sentido aluden a valores y formación integral, el 9\% opina que el área psicológica le aportó elementos necesarios para trabajar, así como el 3\% considera que la carrera le ofreció conocimientos básicos que les han sido de utilidad y solo el $1 \%$ reconoce que el área sociológica le proporcionó elementos para trabajar.

Estos resultados no sorprenden puesto que como ya lo hemos mencionado se han ofrecido buenas bases didácticas y se han cuidado mucho los contenidos del área filosófica. aunque también no deja de preocupar el porcentaje tan bajo, casi nulo, que obtiene el área sociológica. Estos resultados nos hacen ver la necesidad de una reflexión y revisión de los objetivos y contenidos de dicha área.

Entre los egresados que consideran que les faltó formación para su desempeño laboral está el 29\%, encontramos que de éstos el $24 \%$ requieren conocimientos relacionados con el área empresarial, el 19\% opina que la carrera es muy teórica y que se requieren más actividades prácticas, así como un mayor acercamiento a la realidad laboral, el 16\% considera que le faltan elementos de psicología, el 12\% de didáctica y el 11\% del área logotécnica, aquí se refieren a conocimientos relacionados con la computación, el $2 \%$ considera que le faltaron conocimientos del área sociológica y un 15\% señalaron diferentes aspectos que se ubican en el rubro de otros.

Ya por último la información que nos proporciona la variable formación académica nos deja ver que el 18\% considera la formación que recibió como excelente, el 30\% como muy buena y la mayoná, el 51\%, como buena, sólo el 1\% considera que la formación que recibió en la Universidad Panamenicana es regular. 
Actualmente más de la mitad de los egresados que respondieron, el 53\%, realiza estudios de posgrado y de estos el 69\% continua formándose en la Universidad Panamericana, IPADE, IPCE y en la misma facultad de Pedagogía.

Los resultados del presente estudio aportan datos que nos presentan nuevos retos y elementos de reflexión, entre los planes y las acciones para alcanzarlos está la elaboración de un banco de datos de nuestros egresados puesto que es poco gratificante haber conectado a 106 de 1520, es necesario establecer canales de comunicación para estar más cerca y en contacto contínuo con los egresados.

Quedan aún grandes retos por alcanzar, el principal que todos los egresados consideren su formación excelente, ofrecer elementos prácticos que les lleven a obtener mejores ingresos, revisar el área sociológica puesto que es la que aparece ante los alumnos con poco sentido.

De los resultados que aparecen en relación a lo que opinan que hizo falta en su formación, concretamente en los conocimientos del área empresarial, sociológica y logotécnica, consideramos haberlos cubierto ya en el plan de 1999 al igual que la necesidad de acercar más al alumno a la práctica laboral, puesto que contamos con horas de prácticas en centros de trabajo durante las vacaciones de invierno (enero) y verano (junio - julio).

Nos deja satisfechos el reconocimiento, por parte de nuestros exalumnos, de la formación integral que reciben así como de los principios que adquirieron gracias al área filosófica y también el hecho de que más de la mitad de los egresados que cursan estudios de posgrado lo hagan en la Universidad Panamericana.

Queda, pues, continuar con este estudio en dos años más para conocer los resultados que las nuevas modificaciones han tenido en nuestros egresados.

* Antes Instituto Panamericano de Humanidades (I.P.H)

* Última generación con estudios incorporados a la UNAM 


\section{LA ORIENTACIÓN FAMILIAR COMO EXPERIENCIA PEDAGÓGICA}

Gabriel Castellanos Bernal

LA ORIENTACIÓN FAMILIAR ES EL CONJUNTO DE CONTENIDOS, RECURSOS Y procedimientos para apoyar la tarea educativa en la familia. Es innegable la necesidad de esta orientación en una sociedad que plantea nuevos retos y exigencias a los padres, para quienes son insuficientes unas cuantas nociones de educación general. Ahora ellos necesitan aprender con profundidad conceptos de educación familiar, a fin de mantener la unidad y la superación de quienes integran la familia.

En este artículo presentamos algunos aspectos de la actuación del pedagogo como orientador familiar. Su formación profesional, con enfoque humanista, le permite fundamentar teóricamente el trabajo que realiza con padres de familia. Les ayuda a cumplir el compromiso de educar con responsabilidad a sus hijos, y de crear y mantener un ambiente familiar lo más funcional posible.

\section{INTRO DUCCIÓN}

Los cambios científicos, tecnológicos e ideológicos ocurridos en nuestra época han originado modificaciones importantes en diferentes ámbitos de la sociedad. El impacto se ha dejado sentir especialmente en la familia, donde padre, madre e hijos son principales protagonistas del quehacer educativo.

La sociedad cambia con el advenimiento de ideologías que afectan el pensamiento y la actuación del ser humano. También se pretende que la familia cambie: 0 se adapta o muere. Antes, la familia se basaba más en la autoridad del padre, era 